\title{
170.
}

\section{ON SCHELLBACH'S SOLUTION OF MALFATTI'S PROBLEM.}

[From the Quarterly Mathematical Journal, vol. I. (1857), pp. 222-226.]

THE following elegant solution of Malfatti's Problem as applied to spherical triangles is given by Dr Schellbach (Crelle, t. XLV. (1853), p. 186); for the reason which will be mentioned I have made a change of notation.

In a spherical triangle $A B C$ to describe three small circles, each of them touching the other two, and also two sides of the triangle.

Let the sides of the triangle be $a, b, c$, and let $x, y, z$, be the distances of the points of contact from the adjacent angles of the triangle. Then writing

whence

$$
\begin{gathered}
a+b+c=2 s, \\
a-\frac{1}{2} s=l, b-\frac{1}{2} s=m, c-\frac{1}{2} s=n,
\end{gathered}
$$

and putting also

$$
l+m+n=\frac{1}{2} s
$$

$$
\frac{1}{2} s-x=\xi, \frac{1}{2} s-y=\eta, \frac{1}{2} s-z=\zeta,
$$

it is easy to obtain

$$
\left\{\begin{array}{l}
\frac{\cos l \cos \eta \cos \zeta}{\cos \frac{1}{2} s}-\frac{\sin l \sin \eta \sin \zeta}{\sin \frac{1}{2} s}=1 \\
\frac{\cos m \cos \zeta \cos \xi}{\cos \frac{1}{2} s}-\frac{\sin m \sin \zeta \sin \xi}{\sin \frac{1}{2} s}=1 \\
\frac{\cos n \cos \xi \cos \eta}{\cos \frac{1}{2} s}-\frac{\sin n \sin \xi \sin \eta}{\sin \frac{1}{2} s}=1
\end{array}\right.
$$

from which equations the unknown quantities $\xi, \eta, \zeta$, are to be determined. And the equations may be solved without assuming the existence of the relation $l+m+n=\frac{1}{2} s$. 
To solve the equations, let the subsidiary angles $\lambda, \mu, \nu$, be determined by the conditions

$$
\left\{\begin{array}{l}
\frac{\cos \lambda \cos m \cos n}{\cos \frac{1}{2} s}+\frac{\sin \lambda \sin m \sin n}{\sin \frac{1}{2} s}=1, \\
\frac{\cos \mu \cos n \cos l}{\cos \frac{1}{2} s}+\frac{\sin \mu \sin n \sin l}{\sin \frac{1}{2} s}=1, \\
\frac{\cos \nu \cos l \cos m}{\cos \frac{1}{2} s}+\frac{\sin \nu \sin l \sin m}{\sin \frac{1}{2} s}=1,
\end{array}\right.
$$

then it may be shown that

$$
\left\{\begin{array}{l}
\cos (\eta+\zeta)=\frac{\cos \frac{1}{2}(s+\lambda-\zeta)}{\cos \frac{1}{2}(\lambda+l)}, \cos (\eta-\zeta)=\frac{\cos \frac{1}{2}(s-\lambda+l)}{\cos \frac{1}{2}(\lambda+l)} \\
\cos (\zeta+\xi)=\frac{\cos \frac{1}{2}(s+\mu-m)}{\cos \frac{1}{2}(\mu+m)}, \cos (\zeta-\xi)=\frac{\cos \frac{1}{2}(s-\mu+m)}{\cos \frac{1}{2}(\mu+m)} \\
\cos (\xi+\eta)=\frac{\cos \frac{1}{2}(s+\nu-n)}{\cos \frac{1}{2}(\nu+n)}, \cos (\xi-\eta)=\frac{\cos \frac{1}{2}(s-\nu+n)}{\cos \frac{1}{2}(\nu+n)}
\end{array}\right.
$$

If we write

then

$$
\begin{aligned}
& \tan \phi=\tan m \tan n \cot \frac{1}{2} s, \\
& \tan \chi=\tan n \tan l \cot \frac{1}{2} s, \\
& \tan \psi=\tan l \tan m \cot \frac{1}{2} s,
\end{aligned}
$$

$$
\begin{aligned}
& \cos (\lambda-\phi)=\frac{\cos \frac{1}{2} s \cos \phi}{\cos m \cos n}, \\
& \cos (\mu-\chi)=\frac{\cos \frac{1}{2} s \cos \chi}{\cos n \cos l}, \\
& \cos (\nu-\psi)=\frac{\cos \frac{1}{2} s \cos \psi}{\cos l \cos m},
\end{aligned}
$$

equations which give the values of $\lambda, \mu, \nu$, from which $\xi, \eta, \zeta$ are determined as above.

If we suppose that the sides become indefinitely small, we have the case of a plane triangle, and the equations then are

$$
\begin{aligned}
& \eta^{2}+\zeta^{2}+\frac{4 l}{s} \eta \zeta=\frac{1}{4} s^{2}-l^{2}, \\
& \zeta^{2}+\xi^{2}+\frac{4 m}{s} \zeta \xi=\frac{1}{4} s^{2}-m^{2}, \\
& \xi^{2}+\eta^{2}+\frac{4 n}{s} \xi \eta=\frac{1}{4} s^{2}-n^{2}
\end{aligned}
$$

We have here

$$
\begin{aligned}
& (\eta+\zeta)^{2}=\frac{1}{4}\left\{(s+\lambda-l)^{2}-(\lambda+l)^{2}\right\}=\left(\frac{1}{2} s+\lambda\right)\left(\frac{1}{2} s-l\right), \\
& (\eta-\zeta)^{2}=\frac{1}{4}\left\{(s-\lambda+l)^{2}-(\lambda+l)^{2}\right\}=\left(\frac{1}{2} s-\lambda\right)\left(\frac{1}{2} s+l\right),
\end{aligned}
$$


and consequently

where if

$$
\eta^{2}+\zeta^{2}=\frac{1}{2} s^{2}-2 \lambda l, \quad \eta \zeta=s(\lambda-l)
$$

$$
\phi=\frac{2 m n}{s},(\lambda-\phi)^{2}=\frac{1}{4} s^{2}+\phi^{2}-m^{2}-n^{2},
$$

i.e.

Hence

$$
\begin{aligned}
\lambda & =\frac{2 m n}{s}+\sqrt{\frac{1}{4} s^{2}+\frac{4 m^{2} n^{2}}{s^{2}}-m^{2}-n^{2}} \\
& =\frac{2 m n}{s}+\frac{1}{2 s} \sqrt{\left(s^{2}-4 m^{2}\right)\left(s^{2}-4 n^{2}\right)}
\end{aligned}
$$

$$
\begin{aligned}
\eta^{2}+\zeta^{2} & =\frac{1}{2} s^{2}-\frac{4 l m n}{s}-\frac{l}{s} \sqrt{s^{2}-4 m^{2}} \sqrt{s^{2}-4 n^{2}} \\
\eta \zeta & =2 m n-s l+\frac{1}{2} \sqrt{s^{2}-4 m^{2}} \sqrt{s^{2}-4 n^{2}} \\
\zeta^{2}+\xi^{2} & =\frac{1}{2} s^{2}-\frac{4 l m n}{s}-\frac{m}{s} \sqrt{s^{2}-4 n^{2}} \sqrt{s^{2}-4 l^{2}} \\
\zeta \xi & =2 n l-s m+\frac{1}{2} \sqrt{s^{2}-4 n^{2}} \sqrt{s^{2}-4 l^{2}} \\
\xi^{2}+\eta^{2} & =\frac{1}{2} s^{2}-\frac{4 l m n}{s}-\frac{n}{s} \sqrt{s^{2}-4 l^{2}} \sqrt{s^{2}-4 m^{2}} \\
\xi \eta & =2 l m-s n+\frac{1}{2} \sqrt{s^{2}-4 l^{2}} \sqrt{s^{2}-4 m^{2}}
\end{aligned}
$$

which is in fact at once deducible from the formulæ in my paper "On a System of Equations connected with Malfatti's Problem and on another Algebraical System," (Camb. and Dubl. Math. Journ. t. IV. (1849), p. 270 [79]).

Write now for $l, m, n, \xi, \eta$, $\zeta$, their values in terms of $a, b, c, x, y, z$. We have

$$
\left(\frac{1}{2} s-y\right)^{2}+\left(\frac{1}{2} s-z\right)^{2}-\frac{4}{s}\left(\frac{1}{2} s-a\right)\left(\frac{1}{2} s-y\right)\left(\frac{1}{2} s-z\right)=\frac{1}{4} s^{2}-\left(\frac{1}{2} s-a\right)^{2},
$$

i.e.

or reducing

$$
y^{2}+z^{2}-\frac{4}{s}\left(\frac{1}{2} s-a\right) y z-2 a(y+z)+a^{2}=0
$$

$$
(y+z-a)^{2}-4\left(1-\frac{a}{s}\right) y z=0,
$$

and we have thus the system

$$
\begin{aligned}
& y+z+2 \sqrt{1-\frac{a}{s}} \sqrt{y z}=a, \\
& z+x+2 \sqrt{1-\frac{b}{s}} \sqrt{z x}=b, \\
& x+y+2 \sqrt{1-\frac{c}{s}} \sqrt{x y}=c,
\end{aligned}
$$


which are given by Schellbach in the same volume, p. 29; and it was for the sake of facilitating the comparison that the notation has been altered in the case of the spherical triangle. To solve the system, Schellbach writes

reducing the equations to

$$
a=s \sin ^{2} \phi, b=s \sin ^{2} \chi, c=s \sin ^{2} \psi,
$$

whence, putting

$$
\begin{aligned}
& y+z+2 \sqrt{y z} \cos \phi=s \sin ^{2} \phi, \\
& z+x+2 \sqrt{z x} \cos \chi=s \sin ^{2} \chi \\
& x+y+2 \sqrt{x y} \cos \psi=s \sin ^{2} \psi,
\end{aligned}
$$

the equations are satisfied by

$$
\phi+\chi+\psi=2 \sigma,
$$

$$
x=s \sin ^{2}(\sigma-\phi), y=s \sin ^{2}(\sigma-\chi), z=s \sin ^{2}(\sigma-\psi),
$$

which leads to a simple geometrical construction. And if we substitute for $\phi, \chi, \psi, \sigma$, their values, it is easy to obtain

$$
\begin{aligned}
& x=\frac{1}{2} s\left\{1-\sqrt{\left(1-\frac{a}{s}\right)\left(1-\frac{b}{s}\right)\left(1-\frac{c}{s}\right)}\right. \\
& \left.+\sqrt{1-\frac{a}{s}} \sqrt{\frac{\bar{b}}{s}} \sqrt{\frac{c}{s}}-\sqrt{1-\frac{b}{s}} \sqrt{\frac{\bar{c}}{s}} \sqrt{\frac{a}{s}}-\sqrt{1-\frac{c}{s}} \sqrt{\frac{a}{s}} \sqrt{\frac{b}{s}}\right\}, \\
& y=\frac{1}{2} s\left\{1-\sqrt{\left(1-\frac{a}{s}\right)\left(1-\frac{b}{s}\right)\left(1-\frac{c}{s}\right)}\right. \\
& \left.-\sqrt{\left(1-\frac{a}{s}\right)} \sqrt{\frac{b}{s}} \sqrt{\frac{c}{s}}+\sqrt{1-\frac{b}{s}} \sqrt{\frac{c}{s}} \sqrt{\frac{a}{s}}-\sqrt{1-\frac{c}{s}} \sqrt{\frac{a}{s}} \sqrt{\frac{b}{s}}\right\}, \\
& z=\frac{1}{2} s\left\{1-\sqrt{\left(1-\frac{a}{s}\right)\left(1-\frac{b}{s}\right)\left(1-\frac{c}{s}\right)}\right. \\
& \left.-\sqrt{1-\frac{a}{s}} \sqrt{\frac{b}{s}} \sqrt{\frac{c}{s}}-\sqrt{1-\frac{b}{s}} \sqrt{\frac{c}{s}} \sqrt{\frac{a}{s}}+\sqrt{1-\frac{c}{s}} \sqrt{\frac{a}{s}} \sqrt{\frac{b}{s}}\right\}, \\
& y z=\frac{1}{2} s\left\{\sqrt{1-\frac{b}{s}} \sqrt{1-\frac{c}{s}}-\sqrt{1-\frac{a}{s}}+\sqrt{\frac{b}{s}} \sqrt{\frac{c}{s}}\right\}, \\
& z x=\frac{1}{2} s\left\{\sqrt{1-\frac{c}{s}} \sqrt{1-\frac{a}{s}}-\sqrt{1-\frac{b}{s}}+\sqrt{\frac{c}{s}} \sqrt{\frac{a}{s}}\right\}, \\
& x y=\frac{1}{2} s\left\{\sqrt{1-\frac{a}{s}} \sqrt{1-\frac{b}{s}}-\sqrt{1-\frac{c}{s}}+\sqrt{\frac{a}{s}} \sqrt{\frac{b}{s}}\right\},
\end{aligned}
$$

values which are also at once obtained from the formula in my paper above referred to. It may be remarked that the above equations for the determination of $x, y, z$ (the distances of the points of contact from the adjacent angles of the triangle) are very similar in form to those given in the same paper for the determination of $X, Y, Z$, the radii of the inscribed circles. 